\title{
The Biogenesis of Endophilin B1 Containing Vesicles
}

\author{
Yan Chen ${ }^{1}$, Jinhui $\mathrm{Li}^{1}$, Joachim D. Mueller ${ }^{1}$, Barbara Barylko ${ }^{2}$, and Joseph P. Albanesi ${ }^{2}$ \\ ${ }^{1 .}$ Physics Department, University of Minnesota, 116 Church ST. S.E., Minneapolis, MN, 55455 \\ 2. Pharmacology Department, UT Southwestern Medical Center, Dallas, TX, 75239.
}

Fluctuations of fluorescent light emerging from a small region of the sample were first considered more than four decades ago [1]. The technique is known as fluorescence correlation spectroscopy (FCS) and has been extended over the years to study a variety of processes. Here, we focus on a particular type of analysis that is sensitive to the stoichiometry of the complexes, namely brightness analysis. Brightness analysis and its related techniques are used to extract the concentration of the fluorescent complexes and their brightness ("the intensity of a single particle") from the distribution of photon counts. Brightness is a unique parameter that relies on the single molecule sensitivity of FFS and provides a direct quantification of the oligomeric status of populations of GFP-tagged proteins. Since EGFP is a monomer with distinct fluorescence intensity and recombinant 2x-EGFP elicits twice the intensity, the overall brightness of a GFP-tagged protein is a direct function of its oligomeric state (Fig. 1). Thus, brightness analysis is a robust parameter for determining the stoichiometry of protein oligomerizations.

Our studies seek the utility of FFS in the characterization of small cytoplasmic complexes, such as interorganelle transport vesicles, which are below the limit of resolution of conventional live-cell imaging approaches. The mobility of intracellular vesicles is higher because the vesicles are in an aqueous environment, not on a membrane sheet. Whereas commonly used techniques, such as imaging, have limited ability to reveal the physical characteristics of intracellular vesicles, FFS is ideally suited to study such vesicles. The fluctuation amplitude increases with the decrease of sample concentration and increases with the increase of brightness per particle. Intracellular vesicles are at low concentration and each vesicle has multiple copies of a particular protein, which gives rise a high signal-to-noise ratio in fluctuation amplitude, a parameter that is absolutely crucial for quantitative fluorescence fluctuation spectroscopic measurements.

The concentration of vesicles is determined by the brightness spike analysis [2]. The number of brightness spikes at a given data acquisition time, Spike Count Rate, is related to the sample concentration. Figure 2 displays the brightness spikes as a function of fluorescence sphere concentration. The Spike Count Rate was linear at low concentrations (below 100pm), but deviated from linearity at high concentrations. The non-linearity is due to the multiple events occurring very close in time at high concentrations. Nevertheless, even at high concentration, the Spike Count Rate still increases as a function of concentration. Spike Count Rate is therefore a suitable indicator of the sample concentration of rare but bright species.

The brightness spike analysis was used to study endophilin B1 (EndoB1), a protein believed to be important for inducing membrane curvature. The Spike Count Rates of EndoB1 samples were not zero, but they were also not concentration dependent (Fig. 3). In fact, the Spike Count Rate of EndoB1 scattered at all the concentrations examined, suggesting that the incorporation of EndoB1 into a complex depends on another cellular process, perhaps under the control of limiting cellular cofactors. In such a case it is possible that the amount of these putative cofactors may vary from cell to cell, which will explain the scatter we observe in our data. We also performed a similar brightness spike analysis for 
endophilin A2 (EndoA2), a soluble cytosolic dimeric protein. No brightness spikes were detected and the Spike Count Rate was zero at all EndoA2 concentrations examined (Fig. 3).

The identity of vesicles is often revealed by the nature of their coat proteins. We employed heterospecies partition analysis, which allows detection and quantification of the co-existence of two species within the same complex [3]. We examined the co-mobility of EndoB1 and its putative coat protein by either cotransfecting EndoB1-GFP with Caveolin1-mCherry or with Clathrin-mCherry. The resulting heterospecies brightness vectors are displayed in figure 4. All the heterospecies vectors of EndoB1/Clathrin were scattered along the "green species only line", which indicated that EndoB1-GFP wasn't associated with Clathrin-mCherry on vesicles. The HSP vectors of EndoB1/Caveolin1, on the other hand, were scattered, but with many data in the "green-red co-mobile zone", suggesting that at least a subset of EndoB1 containing vesicles have Caveolin1 as a coat.

In conclusion, we show that multiple copies of endophilin B1 (EndoB1) associate with vesicles coated with caveolin but not clathrin. Our data indicate that intracellular vesicle trafficking is a selective process, and overexpression alone doesn't guarantee that two proteins will co-exist on the same vesicles. By studying the coat, and the concentration of the vesicles, we are able to show the likelihood of the location and mechanisms of the biogenesis of EndoB1 vesicles.

\section{References:}

[1] D. Magde, E. L. Elson, and W. W. Webb, "Fluorescence correlation spectroscopy. II. An experimental realization," Biopolymers, vol. 13, no. 1, pp. 29-61, Jan. 1974.

[2] J. Li, B. Barylko, J. Johnson, J. D. Mueller, J. P. Albanesi, and Y. Chen, "Molecular Brightness Analysis Reveals Phosphatidylinositol 4-Kinase II $\beta$ Association with Clathrin-Coated Vesicles in Living Cells," Biophys. J., vol. 103, no. 8, pp. 1657-1665, Oct. 2012.

[3] B. Wu, Y. Chen, and J. D. Müller, "Heterospecies partition analysis reveals binding curve and stoichiometry of protein interactions in living cells," Proc. Natl. Acad. Sci., vol. 107, no. 9, pp. 4117-4122, Mar. 2010.

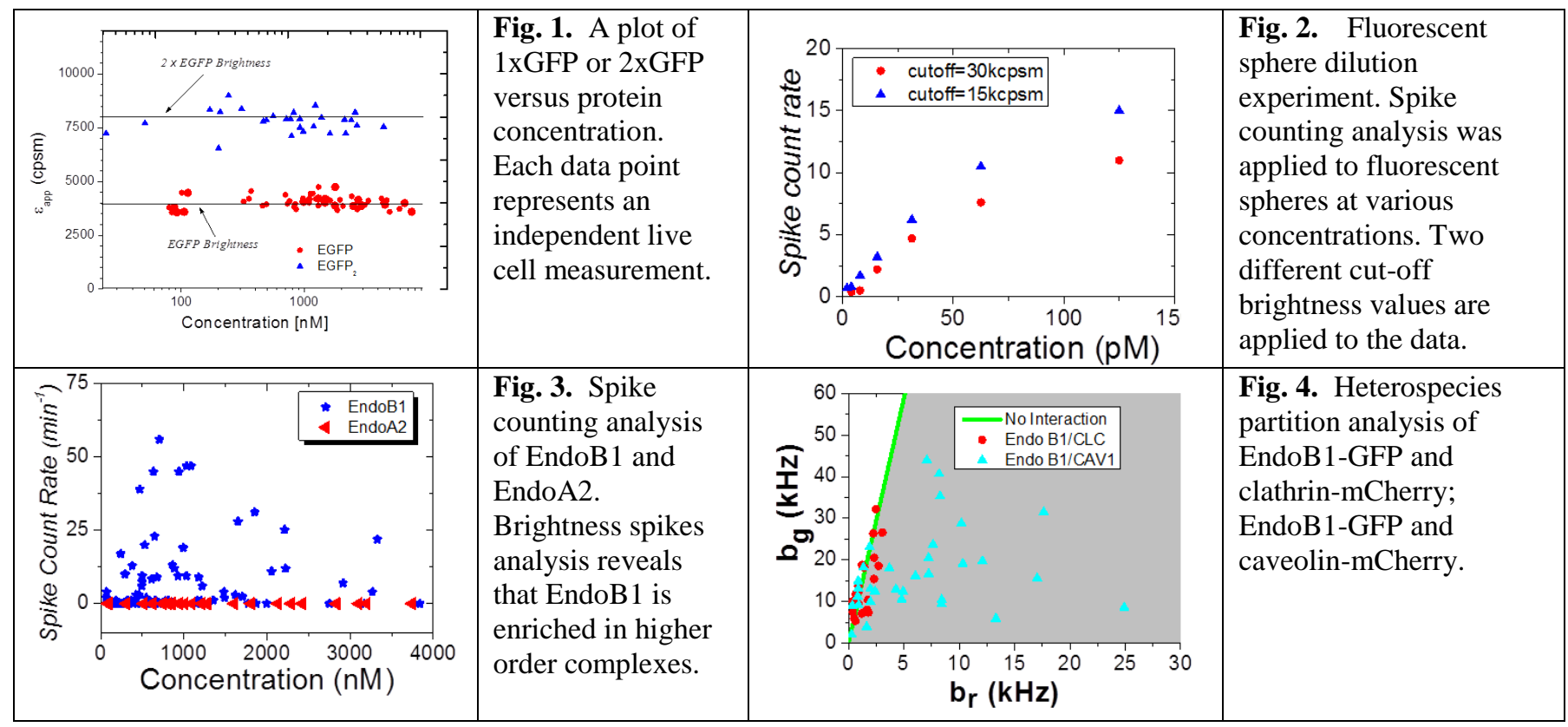

